Wright State University

CORE Scholar

Physics Faculty Publications

Physics

$11-1-1999$

\title{
Low-Frequency Noise in N-GaN with High Electron Mobility
}

M. E. Levinshtein

S. L. Rumyantsev

David C. Look

Wright State University - Main Campus, david.look@wright.edu

Richard J. Molnar

M. A. Khan

See next page for additional authors

Follow this and additional works at: https://corescholar.libraries.wright.edu/physics

Part of the Physics Commons

\section{Repository Citation}

Levinshtein, M. E., Rumyantsev, S. L., Look, D. C., Molnar, R. J., Khan, M. A., Simin, G., Adivarahan, V., \& Shur, M. S. (1999). Low-Frequency Noise in N-GaN with High Electron Mobility. Journal of Applied Physics, 86 (9), 5075-5078.

https://corescholar.libraries.wright.edu/physics/140

This Article is brought to you for free and open access by the Physics at CORE Scholar. It has been accepted for inclusion in Physics Faculty Publications by an authorized administrator of CORE Scholar. For more information, please contact library-corescholar@wright.edu. 
Authors

M. E. Levinshtein, S. L. Rumyantsev, David C. Look, Richard J. Molnar, M. A. Khan, G. Simin, V. Adivarahan, and M. S. Shur

This article is available at CORE Scholar: https://corescholar.libraries.wright.edu/physics/140 


\section{Low-frequency noise in $n$-GaN with high electron mobility}

M. E. Levinshtein and S. L. Rumyantsev

Solid State Electronics Division, The Ioffe Physicotechnical Institute of Russian Academy of Sciences, 194021, St. Petersburg, Russia

D. C. Look

Semiconductor Research Center, Wright State University, Dayton, Ohio 45435

R. J. Molnar

Lincoln Laboratory, Massachusetts Institute of Technology, Lexington, Massachussetts 02173

M. Asif Khan, G. Simin, and V. Adivarahan

Department of Electrical and Computer Engineering, University of South Carolina, Columbia, South Carolina 29208

M. S. Shur ${ }^{\mathrm{a})}$

Department of Electrical, Computer, and Systems Engineering and Center for Integrated Electronics and Electronics Manufacturing, CII 9017, Rensselaer Polytechnic Institute, Troy, New York 12180-3590

(Received 30 June 1999; accepted for publication 3 August 1999)

We report on the results of measurements of low frequency noise in $n$-type gallium nitride ( $\mathrm{GaN}$ ) grown on sapphire with $300 \mathrm{~K}$ electron mobility of $790 \mathrm{~cm}^{2} / \mathrm{V} \mathrm{s}$. The noise spectra have the form of $1 / f$ noise with a Hooge parameter $\alpha$ of approximately $5 \times 10^{-2}$. This value of $\alpha$ is two orders of magnitude smaller than that observed before in $n-\mathrm{GaN}$. The obtained results show that the level of flicker noise in $\mathrm{GaN}$, just like that in GaAs and $\mathrm{Si}$, strongly depends on the structural perfection of the material (the amplitude of the $1 / f$ noise is much smaller in material with high mobility). The effects of band-to-band illumination on the low-frequency noise show that $1 / f$ noise in GaN might be caused by the occupancy fluctuations of the tail states near the band edges. This mechanism of the $1 / f$ noise is similar to that in GaAs and $\mathrm{Si}$. (C) 1999 American Institute of Physics. [S0021-8979(99)07521-0]

\section{INTRODUCTION}

Gallium nitride $(\mathrm{GaN})$ has an excellent potential for high temperature, high frequency, and high power microwave applications. Blue and violet light emitting diodes based on GaN have been commercialized, and GaN-based lasers, UV visible-blind photodetectors, and piezoelectric sensors have also been demonstrated (see, for example, Refs. 1-3). The level of the low-frequency noise is one of the most important parameters, which determines whether the devices are suitable for microwave and optical communication systems. First estimates of the low-frequency noise level in $n$-GaN were made using visible-blind $\mathrm{GaN} p$ - $n$ junction photodetectors. ${ }^{4,5}$ The estimated $\alpha$ value was very high ( $\alpha$ $\approx 3$ ). This value is comparable with the values of $\alpha$ for such disordered materials as conducting polymers. ${ }^{6}$ The results obtained for $n$-GaN resistors confirmed these data. ${ }^{7,8}$

One might suggest several possible reasons for such a large $1 / f$-noise level. The level of the $1 / f$ noise is much higher for a semiconductor material with imperfections (see, for example, Ref. 9). Among other factors, a high dislocation density strongly increases the level of $1 / f$ noise in certain cases. ${ }^{10,11}$ The measured dislocation density in GaN samples grown on sapphire is on the order of $10^{9}-10^{10} \mathrm{~cm}^{-2} \cdot{ }^{12}$ On the other hand, theory ${ }^{9,13}$ predicts that the level of $1 / f$ noise should be proportional to the density of the tail states near

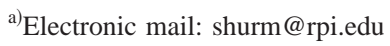

the band edges. (It is worth noting that the density of states in the band tails depends also on the structural perfection of the material). ${ }^{14,15}$ The density of states in the conduction band tail in $\mathrm{GaN}$ is much higher than that for $\mathrm{Si}$ and $\mathrm{GaAs}$ (see, for example, Ref. 16).

Structural perfection of a semiconductor is often estimated based on the values of the low-field carrier mobility. For the samples used in Refs. 7 and 8, the electron mobility $\mu_{n}$ was approximately $60 \mathrm{~cm}^{2} / \mathrm{V} \mathrm{s}$ and was practically temperature independent in the range between 77 and $400 \mathrm{~K}$.

In this article, we report on the results of the measurements of the low-frequency noise in the samples grown on sapphire substrates with $\mu_{n}=790 \mathrm{~cm}^{2} / \mathrm{V} \mathrm{s}$ at $300 \mathrm{~K}$, and with the temperature dependence $\mu_{n}(T)$ close to that predicted by theory. ${ }^{17}$

\section{EXPERIMENTAL DETAILS}

The 20- $\mu \mathrm{m}$-thick sample was grown by hydride vapor phase epitaxy on sapphire. Sputtered $\mathrm{ZnO}$ was used as a buffer layer between the sapphire and $\mathrm{GaN}$, but the $\mathrm{ZnO}$ was no longer present after completion of the growth. ${ }^{18}$ Except for a highly defective interface region of a few thousand angstroms thickness, the sample was of very high quality, with a $300 \mathrm{~K}$ mobility of $790 \mathrm{~cm}^{2} / \mathrm{V} \mathrm{s}$, and a carrier concentration of $1.28 \times 10^{17} \mathrm{~cm}^{-3}$. Fits to the temperaturedependent Hall data in the bulk region yielded donor and acceptor concentrations of $2.1 \times 10^{17}$ and $5 \times 10^{16} \mathrm{~cm}^{-3}$, re- 


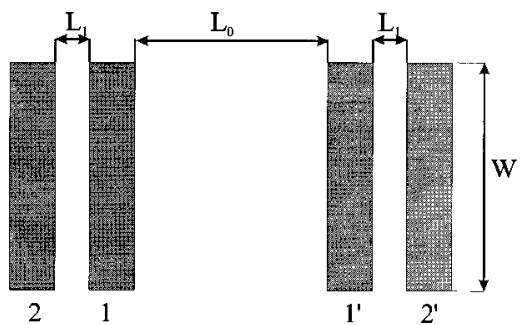

FIG. 1. Top view of the sample geometry. $L_{0}=1100, L_{1}=80, W$ $=250 \mu \mathrm{m}$, the thickness of the film $t=20 \mu \mathrm{m}$.

spectively, and a donor activation energy of $16 \mathrm{meV}$. The interface region, on the other hand, was strongly degenerate, with a sheet carrier concentration of $8 \times 10^{14} \mathrm{~cm}^{-2}$ and an electron mobility of $55 \mathrm{~cm}^{2} / \mathrm{V}$ s. Further information on the electrical properties can be found in Ref. 19. Ti $(54 \AA)-\mathrm{Al}$ $(1920 \AA)$ contacts were deposited on the surface of the film and annealed at a temperature of $550^{\circ} \mathrm{C}$ for $2 \mathrm{~min}$. The contact resistance, $R_{c}$, has been estimated using a transmission line model measurement. ${ }^{20}$

Low-frequency noise was measured between contacts 1 and 2, and 1 and $1^{\prime}$ (see Fig. 1) in the dark and under bandto-band illumination. Current-voltage characteristics measured between the contacts were linear and symmetrical with an accuracy of approximately $1 \%$.

Resistance $R_{12}$ between contacts 1 and 2 was equal to $9.85 \Omega$; resistance $R_{11}$, between the contacts 1 and $1^{\prime}$ was equal to $30.2 \Omega$. The estimated contact resistance was approximately $1.9 \Omega$ for both configurations.

\section{RESULTS AND DISCUSSIONS}

Figure 2 shows the frequency dependencies of the noise relative spectral density measured between the contacts 1 and 2. In the dark (curve 1), the noise is typical $1 / f$ (flicker) noise.

The flicker noise level in different materials is frequently characterized by the dimensionless Hooge parameter, $\alpha^{21}$

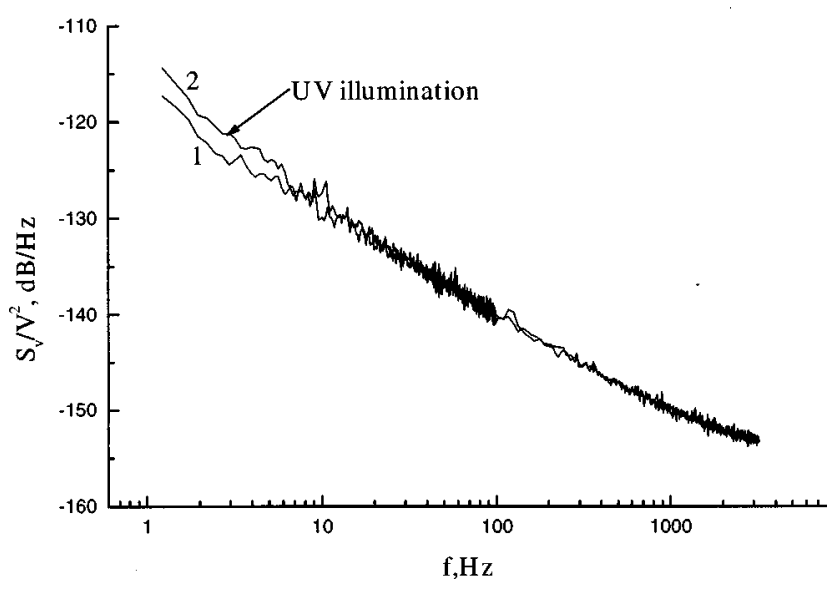

FIG. 2. Frequency dependencies of the current noise relative spectral density $S_{\mathrm{v}} / V^{2}$ in the dark (curve 1) and under band-to-band illumination (curve 2) measured between contacts 1 and 2 .

$$
\alpha=\frac{S_{V}}{V_{o}^{2}} f N,
$$

where $N=L_{1} \times W \times t \times n_{0}$ is the total number of the conduction electrons in the sample (determined for the homogeneous situation as the value of the carrier density times the sample volume), $f$ is the frequency, $V_{o}=I R_{12}$ is the voltage drop between contacts. The value of $\alpha$ calculated accordingly for the data presented in Fig. 2 is equal to $\sim 5$ $\times 10^{-2}$. This value is two orders of magnitude smaller than values of $\alpha$ in $n$-GaN reported earlier. ${ }^{4,5,8}$ Nevertheless, even this relatively low value of $\alpha$ is overestimated for two reasons.

First, as usual for two-probe measurements, the contacts can give an essential contribution to the overall lowfrequency noise. ${ }^{22}$ Second, the obtained estimate is valid for a fully homogeneous current density distribution. However, in our case, the contacts penetrate into the GaN film not more than for a fraction of a micron. Estimated transfer length is $L_{T}=(6-8) \mu m$. Therefore, the current density $\mathrm{j}$ close to and under the contacts is substantially larger than the average current density across the sample. The noise spectral density $S_{V}$ strongly depends on j: ${ }^{21}$

$$
S_{V}=\frac{1}{\mathrm{I}^{2}} \iiint \frac{\alpha \rho^{2} j^{4}}{n f} d \mathrm{v},
$$

where $\rho=1 / \sigma$ is the local resistivity, and $n$ is the local electron concentration. The integral in Eq. (2) should be taken over the whole sample. However due to very strong dependence of $S_{V}$ on $\mathrm{j}$, the effective value of the total number of conduction electrons $N$ in Eq. (1) (and, therefore, the value of $\alpha$ ) should be less than the value of $N=L \times W \times t \times n_{0}$ used in the estimate for a homogeneous case. In fact, a relatively small region close to the contacts could make a dominant contribution to the $1 / f$ noise.

Curve 2 in Fig. 2 presents the results under band-to-band illumination with an incandescent lamp. At a given illumination intensity, the photoconductivity $\sigma / \sigma_{0} \approx 2 \times 10^{-2}$. The effect of the illumination is relatively weak. However the qualitative effect is quite similar to that for $\mathrm{Si}$ and GaAs. ${ }^{9,23}$ The illumination has no effect at higher frequencies and increases the noise at relatively low frequencies.

Figure 3 shows the frequency dependencies of the noise relative spectral density measured between the contacts 1 and $1^{\prime}$. The curve measured in the dark (curve 1) has the form of $1 / f$ noise (flicker) noise. Comparing curves 1 in Figs. 2 and 3 one can see that the difference in the level of the dark noise is rather small for these two very different electrode configurations. The noise measured between contacts 1 and $1^{\prime}$ should be considerably less than that between contacts 1 and 2. It is clear that the total number of the conduction electrons involved is substantially smaller for the case represented in Fig. 2 (contacts 1 and 2) compared to that shown in Fig. 3 (contacts 1 and $1^{\prime}$ ) provided the metal contacts penetrated the whole depth of the film.

A crude estimate for the expected noise level measured between contacts 1 and 1' can be obtained using the results of Ref. 24. According to these results, if the distance between the contacts $L_{0}$ is much larger than the width of the 


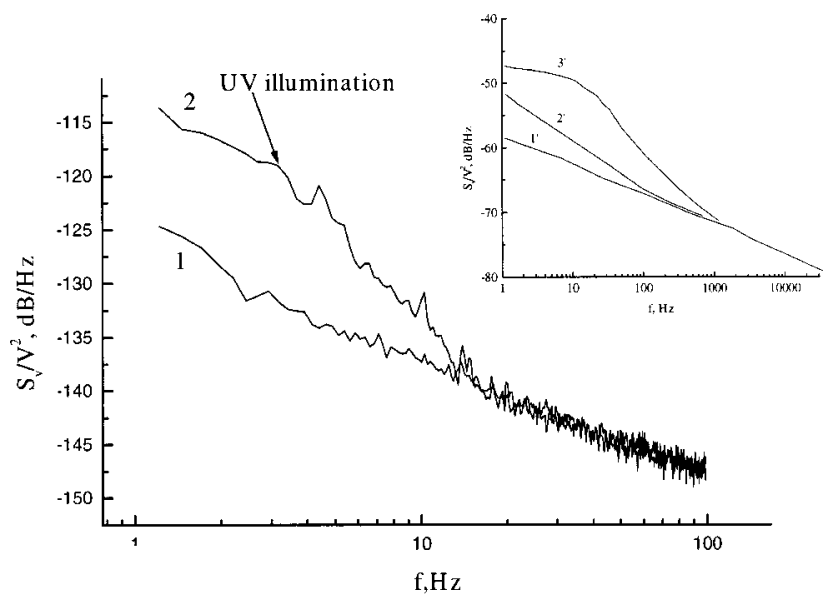

FIG. 3. Frequency dependencies of the current noise relative spectral density $S_{\mathrm{v}} / V^{2}$ in the dark (curve 1) and under band-to-band illumination (curve 2) measured between contacts 1 and $1^{\prime}$. Inset shows the theoretical frequency dependencies of the noise spectral density under band-to-band illumination, which changes the occupancy of the tail levels responsible for the $1 / f$ noise. $1^{\prime} \cdot b=0,2^{\prime} \cdot b=10^{-16} \cdot 3^{\prime} \cdot b=10^{-15}$. Here $b$ is a parameter which is approximately proportional to the illumination intensity (see Ref. 23).

contacts $W\left(L_{0} \gg W\right.$, see Fig. 1$)$, the rectangular contacts can be approximated by circular contacts with the effective radius $r_{\text {eff }}=W / 4$. For such contacts (at $L_{0} \gg W$ ) the integral (2) can be evaluated to yield

$$
\begin{aligned}
S_{V} & =\frac{1}{I^{2}} \iiint \frac{\alpha \rho^{2} j^{4}}{n f} d \mathrm{v} \\
& =\frac{\alpha \times t \times \sigma^{2}}{n_{0} \times f \times I^{2}} \int E^{4} d s \\
& =\frac{\alpha \times V_{0}^{2}}{16 \pi^{2} \times t \times n_{0} \times f \times r_{\mathrm{eff}}^{2} \times \ln ^{2}\left(\frac{2 L_{0}}{r_{\text {eff }}}\right)},
\end{aligned}
$$

where $t$ is the thickness of the film, $r_{\text {eff }}=W / 4$ (see Fig. 1), and $V_{0}$ is the bias applied between the circular contacts. Hence,

$$
\alpha=\frac{S_{V}}{V_{0}^{2}} \times f \times 16 \pi^{2} \times n_{0} \times r_{\mathrm{eff}}^{2} \times t \times \ln ^{2}\left(\frac{2 L_{0}}{r_{\mathrm{eff}}}\right) .
$$

Comparing Eqs. (1) and (4) with $t=20 \mu \mathrm{m}, r_{\text {eff }}=60 \mu \mathrm{m}$, and $L_{0}=1100 \mu \mathrm{m}$, one can conclude that the flicker noise measured between contacts 1 and $1^{\prime}$ at the same frequency $f$ should be $\approx 25 \mathrm{~dB}$ smaller. However, the measured difference in the noise levels is only $\approx 7 \mathrm{~dB}$ (compare curves 1 in Figs. 2 and 3).

Once again, these results demonstrate that relatively small regions close to the surface contacts disproportionally contribute to the noise, and the deduced value of $\alpha \approx 5$ $\times 10^{-2}$ is only an upper bound for $\alpha$.

Curve 2 in Fig. 3 represents the results under band-toband illumination. At a given illumination intensity, the photoconductivity $\sigma / \sigma_{0} \approx 5 \times 10^{-3}$. Despite the fact that this photoconductivity is less than that for the case represented in Fig. 2, the effect of the band-to-band illumination is much stronger. Qualitatively, the curves 2 in Figs. 2 and 3 are very similar: the illumination has no effect at the higher frequencies of analysis and increases the noise at relatively low frequencies.

Such an effect of band-to-band illumination on the $1 / f$ noise was analyzed in detail for Si samples in Ref. 25 using the model of $1 / f$ noise developed in Ref. 13 (see the inset in Fig. 3). Based on the multiphonon capture mechanism, ${ }^{26}$ the model predicts that the capture time constant $\tau$ exponentially increases with the energy of the levels in the tail of density of states, $E(E=0$ at the conduction band boundary)

$$
\tau(E)=\tau_{0} \times \exp \left(E / E_{1}\right) .
$$

Here $E_{1}$ is a characteristic energy of the capture crosssection reduction. (For $\mathrm{Si}, E_{1}=5 \mathrm{meV} ;{ }^{25}$ for $\mathrm{GaAs}, E_{1}$ $=10 \mathrm{meV}$. $)^{9}$

The holes generated as a result of band-to-band illumination are captured by the tail states. This process is accompanied by changes in the level occupancy and, hence, by changes in the noise generated by the tail levels of energy $E$. The probability of hole capture by the tail states is independent of energy $E$. However, the level occupancy under steady state conditions very strongly depends on $E$ because the electron capture time $\tau$ for the empty levels exponentially depends on energy [see Eq. (5)].

Weak illumination has practically no effect on the level occupancy for the levels at low energy, E. Such levels are responsible for the $1 / f$ noise at relatively high frequencies because the electron capture time for these levels is rather small.

The levels with large energies are practically fully emptied even at very low illumination intensities, since the electron capture time for these levels is exponentially large. Such "deep" levels are responsible for the noise at the very low frequencies. Therefore, one can expect that at very low frequencies the spectral density of noise is frequency independent (see curve 3 in the inset). Such an effect has been observed in $\mathrm{Si}$ and GaAs. ${ }^{9}$

At intermediate frequencies (i.e., for the levels with intermediate energies, $E$ ), the holes captured by the tail levels reduce the level occupancy, $F(F \approx 2 / 3$ for the maximum noise level-generated noise).

\section{CONCLUSIONS}

The comparison of the observed effect of band-to-band illumination on the low-frequency noise (see Fig. 3) with the theory (see the inset) shows that the nature of the $1 / f$ noise in GaN should be similar to that in GaAs and Si. The $1 / f$ noise is caused by the fluctuations of the occupancy of the tail states near the band edges.

\section{ACKNOWLEDGMENTS}

The work at Lincoln Laboratory was sponsored by the United States Air Force under Air Force Contract No. F19628-95-C-0002. Opinions, interpretations, conclusions, and recommendations are those of the authors and are not necessarily endorsed by the United States Air Force. The 
work at Rensselaer Polytechnic Institute was partially supported by the Office of Naval Research (Project Monitor Dr. Colin Wood).

${ }^{1}$ S. J. Pearton and C. Kuo, Mater. Res. Bull. 22, 17 (1997).

${ }^{2}$ S. Nakamura, Mater. Res. Bull. 22, 29 (1997).

${ }^{3}$ R. Gaska, J. Yang, A. D. Bykhovski, Michael S. Shur, V. V. Kaminski, and S. M. Soloviov, Appl. Phys. Lett. 71, 3817 (1997).

${ }^{4}$ A. Osinsky, S. Gangopadhyay, R. Gaska, B. Williams, M. A. Khan, D. Kusenkov, and H. Temkin, Appl. Phys. Lett. 71, 2334 (1997).

${ }^{5}$ D. V. Kusenkov, H. Temkin, A. Osinsky, R. Gaska, and M. Khan, J. Appl. Phys. 83, 2142 (1998).

${ }^{6}$ E. G. Guk, M. E. Levinshtein, V. A. Marikhin, L. P. Myasnikova, and S. L. Rumyantsev, Phys. Solid State 39, 690 (1997).

${ }^{7}$ N. V. Dyakonova, M. E. Levinshtein, S. Contreras, W. Knap, B. Beaumont, and P. Gibart, Semiconductors 32, 257 (1998)

${ }^{8}$ N. V. Dyakonova, M. E. Levinshtein, S. L. Rumyantsev, S. Contreras, W. Knap, R. Gaska, and M. S. Shur. 2 Russian Workshop "GaN, InN, and AlN: structures and devices." St. Petersburg, St. Petersburg Technical Univ. Russia, 2 June 1998.

${ }^{9}$ N. V. D’yakonova, M. E. Levinshtein, and S. L. Rumyantsev, Sov. Phys. Semicond. 25, 1241 (1991).

${ }^{10}$ A. M. Svetlichnyi, L. A. Koledov, V. V. Zotov, and E. F. Uvarov, Sov. Phys. Semicond. 14, 345 (1980).

${ }^{11}$ V. M. Vinokur and S. P. Obukhov, Zh. Eksp. Teor. Fiz. 95, 223 (1989) [Sov. Phys. JETP ].

${ }^{12}$ R. Gaska (private communication).
${ }^{13}$ N. V. Dyakonova and M. E. Levinshtein, Sov. Phys. Semicond. 23, 175 (1989).

${ }^{14}$ M. Skowronski, J. Lagowski, M. Milshtein, C. H. Kang, F. P. Dabkowski, A. Hennel, and H. C. Gatos, J. Appl. Phys. 62, 3791 (1987).

${ }^{15}$ M. E. Levinshtein and S. L. Rumyantsev, Sov. Phys. Semicond. 24, 1125 (1990).

${ }^{16}$ O. Ambacher, W. Rieger, P. Ansmann, H. Angerer, T. D. Moustakas, and M. Stutzman, Solid State Commun. 97, 365 (1996).

${ }^{17}$ D. C. Look, Mater. Sci. Eng., B 50, 50 (1997).

${ }^{18}$ R. J. Molnar, K. B. Nichols, P. Makai, E. R. Brown, and I. Melngailis, Mater. Res. Soc. Symp. Proc. 378, 479 (1995).

${ }^{19}$ D. C. Look and R. J. Molnar, Appl. Phys. Lett. 70, 3377 (1997).

${ }^{20}$ G. K. Reeves and H. B. Harrison, IEEE Electron Device Lett. EDL-3, 111 (1982).

${ }^{21}$ F. N. Hooge, T. G. M. Kleinpenning, and L. K. J. Vandamme, Rep. Prog. Phys. 44, 479 (1981).

${ }^{22}$ M. E. Levinshtein, F. Pascal, S. Contreras, W. Knap, S. L. Rumyantsev, R. Gaska, J. W. Jang, and M. S. Shur, Appl. Phys. Lett. 72, 3053 (1998).

${ }^{23}$ E. G. Guk, N. V. D'yakonova, M. E. Levinshtein, and S. L. Rumyantsev, Sov. Phys. Semicond. 24, 513 (1991).

${ }^{24}$ Fr. Ollendorf, Erdstrome. Russian ed. (Fizmatgiz, Moscow, Leningrad, 1932).

${ }^{25}$ N. V. Dyakonova, M. E. Levinshtein, D. L. Plotkin, and S. L. Rumyantsev, Sov. Phys. Semicond. 24, 527 (1990).

${ }^{26}$ V. N. Abakumov, V. I. Perel, and I. N. Yassievich, Nonradiative Recombination in Semiconductors, Modern Problems in Condensed Matter Science, edited by V. M. Agranovich and A. A. Maradudin (North-Holland, Amsterdam, 1991), Vol. 33 